\title{
BEAM Dragster Solar Robot with its Strategic Winning Design Analysis for the Nexus Photoroller Competition
}

\author{
Siddarth Jain \\ Department of Electronics \& Communication \\ Engineering \\ Medi-Caps Institute of Technology \& Management \\ Indore, India
}

\author{
Navneet Tiwari \\ Department of Electronics \& Communication \\ Engineering \\ Medi-Caps Institute of Technology \& Management \\ Indore, India
}

\begin{abstract}
We are introducing a novel methodology that demonstrates the feasibility of the crystal oscillator as a solar engine. The design objective is to construct a solar robotic machine which uses the solar power as the only source of energy to race over $1000 \mathrm{~mm}$ straight track in the minimum time spell. During the design and implementation phase, we have successfully introduced the application of the gold capacitor in the relaxation oscillator. This successful design not only beats Asia's 150 teams but also fetches the mind of the 10,000 techies from all over India.
\end{abstract}

\section{Keywords}

Robotics, Photovore, Solar Engine, Solar Robotics, Touch sensors(Feelers).

\section{INTRODUCTION}

If, we scrutinize various aspects of our life, we will surely find the surrounding of life style with the consumptionconsumption of natural resources and energy. The renewable energy is a vital part of all available energy, which is capable to fulfill our entire energy requirement. Among all available forms of renewable energy the solar energy is clean, green, free and widely available. From over the past century, we have seen a relentless approach toward the procurement of world of automation and ease. The industrial revolution have significantly introduces up various electronic and mechanical devices. In present scenario, we have become dependent over the functioning of these devices for our continued growth and development. More recently the exploitation of the oil, natural gases and other fossil fuels has empowered the wheels of industry and automation. We accept that the automation requires less human input effort but it surely requires a vital part of the energy. Thus, we have enslaved ourselves to non renewable energy sources and created a world whose possibility diminishes in absence of these non-renewable sources. The human beings propensity to push the boundary of the Universe by exploring the deep uncharted realms of space would get mollify by a proper delineation of non renewable energy flow cycle. In present scenario, we need to provide energy for these distant ventures - which is hard with conventional means. The Spirit Mars Rover is a good example of solar robot which is equipped with a 140 Watt array. In our daily life, we always see the increasing penetration of robots into our households - Roomba and Scooba are the household names and represent the readily available domestic robots. However, at present, service robot requires a clumsy recharging stations that perils the possibility of its prevalence. What, if your domestic robots could roam freely around your house and that would be empowered by nothing more than the sun shining through your window and the ambient light freely available in your rooms?

\section{DESIGN CONSIDERATION COMPETITION}

The event 'photovore' inspires us for the development of light seeking solar robot, which follows the desired path under the sun light. Our consistent research efforts in the field of solar robotics motivate us to apply the nitty-gritty of the solar robotics in this direction. In the dream of winning this competition, we have started our efforts in this direction. The upcoming deals with the various issues related to the designing of the solar robots.

\subsection{Solar Cell: Paramount Design Factor:}

The emergence of the solar cell brings tremendous changes in the domestic utilization of the renewable energy. Photovoltaic Effect is the phenomenon that describes the function of the solar cell. A solar cell converts the solar energy in the form of electrical energy. The solar cells can be subdivided into three generation. The first generation technology solar cells are widely adaptable in nature, which accounts around $89.7 \%$ of the net production in the commercial year 2007. Due to the procurement of high quality and large volumetric surface, we have included the 1st Generation solar cells in our design. During the designing phase of the photopper, we concentrated our efforts over the energy utilization of the solar cells. In our design, we have used out polycrystalline solar cells. These cells were epoxy encapsulated and polycrystalline in nature, which were mounted over sturdy fiber glass backing. The color coded polarity can be bifurcated out with the help of solder tabs. These solar cells are come within the $37 \mathrm{X} 33 \mathrm{~mm}$ and $37 \times 66 \mathrm{~mm}$ package [7]. The $37 \mathrm{X} 33 \mathrm{~mm}$ has the voltage $\&$ current. specification of $6.7 \mathrm{~V}$ and $31 \mathrm{~mA}$, which were significantly lower than by 33 X $66 \mathrm{~mm}$ solar cells. These solar cells have the current and voltage specification of $8 \mathrm{~V}$ and $44 \mathrm{~mA}$. But, by proper trimming of $37 \times 33 \mathrm{~mm}$ of the serial bus connector on the back hand, we have found that these solar cells can be altered to provide about $3.3 \mathrm{~V}$ at near $80 \mathrm{~mA}$ (in full sun) by separating the two sides of the cell and then to cross-connect the two sides of the bus to the + and contacts. It would be somehow very nice because it can provide the half of the voltage but twice the current, still all in the same solar package! Along with that, these solar cells are also validated by the National Renewable Energy Laboratory and also compliance with three reliable certifications of solar equipment: - UL, IEC and IEEE (both U.S. standards). All these features are highly supportable and provide a good platform for our design. During competition, we have arranged out all these solar cells in serial fashion which significantly enhance the performance of the rover in arena. 


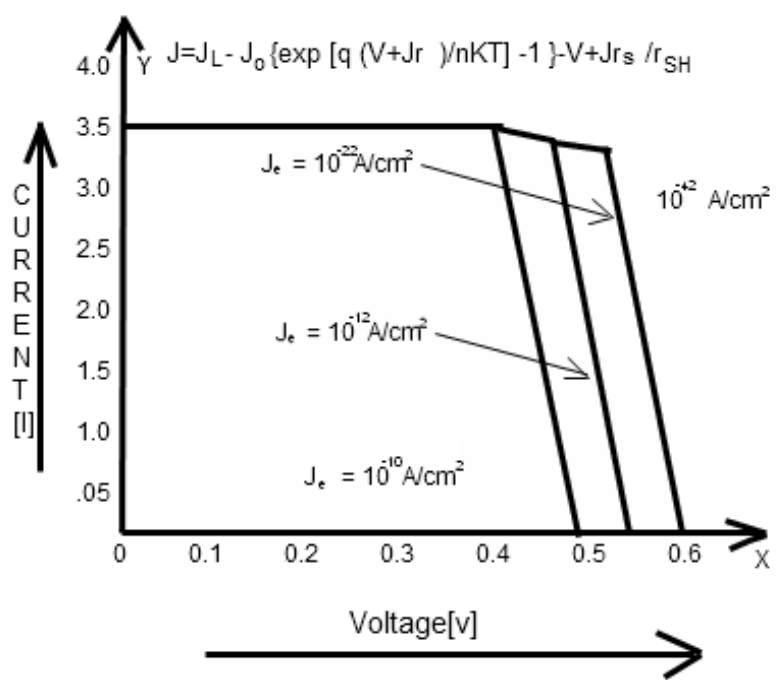

Figure 1. Performance plot between voltage and current of epoxy-crystalline photo-cell.

\subsection{Gold Capacitor:}

The capacitance is the important design parameter that directly affects the performance of the robot in the arena. The volumetric efficiency of the capacitor is a vital and parametric constraint of a solar robot. The Gold Capacitor is an Electric Double Layered Capacitors, which offers the highest volumetric efficiency among all available capacitor technology. In our design, we have used the Panasonic Gold Capacitor because it had a very sharp charging and discharging cycles that is thoroughly unaffected by the number of cycles. [8] This EN Series Capacitor can withstand up to 240 degree Centigrade and its miniature design increases feasibility of the machine [by reducing the weight and size of the robot].The Gold Capacitor doesn't contain any heavy metal and during the charging and discharging there is no expendable chemical reactions takes place.[7] All these features make them multi adaptable and Environmental friendly in nature.

\subsection{Arena of Nexus Central Zone}

A. Objective: The construction of the solar robotic machine which uses the solar power as the only source of energy to race over $1000 \mathrm{~mm}$ straight track in the minimum time spell.

B. Arena Description: The competition is organized under title of the "Photoroller: Student Design Competition". Now, we have to design our robot according to the mandated specification of he arena. The racing strip is composed of two side by side of $1000 \mathrm{~mm}$ lanes of clean, smooth, level, flat ply wood, with $25 \mathrm{~mm}$ high white walls along both sides of each lane. The arena is enlightened by two $500 \mathrm{~W}$ halogen lamps which is $500 \mathrm{~mm}$ above to the track. The light source for the table will start off with the light source directly above them. This light source is to be situated at an acute position to the finish line. The arena is to be situated at the indoor but the separate place is to be offer for the testing of the circuit.

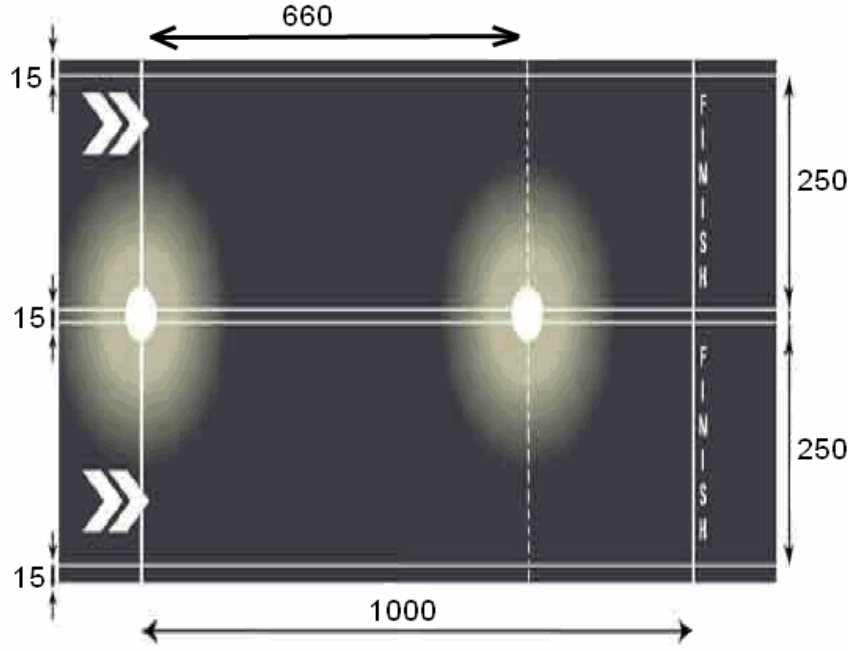

All Dimension are in $\mathrm{mm}$

Figure 2. Demonstration of top view of the arena for the Central Regional Phase Round at SGSITS, Indore

\section{INTERNATIONAL DESIGN: RULES \& REGULATION}

The student design competition consists some parametric constraint that regulates the flow of the design. 17] During our solar robotics design, we follow the guided rules and found that all this constraint is very helpful for the successful accomplishment of the design. This design pushed our efforts in the right direction and fetches the mind of many techies from all over India. These standards help us for the successful accomplishment of the task and left a long lasting trail in the well-known arena of the Central zone (situated at the SGSIT Indore). Now for the better understanding of the event some of the mandated rules have been listed below:

[i.] The machine should be automatically controlled the manual controlling of the robot is not allowed in arena.

[ii.] The physical structure of the robot must be fit within the box of dimension of the $250 \mathrm{~mm} \mathrm{X} 250 \mathrm{~mm}$ X $250 \mathrm{~mm}$. These specifications will be thoroughly checked before the starting of competition.

[iii.] During the performance of the solar robot in the arena. The design could expand its design structure, but it should not violate the rules and regulations of the Arena. The judges hold the right to subjectively determine which machine violates the clause.

[iv.] The readymade mechanism of the machines or readymade assembly gears is not allowed.

[v.] The machine cannot use any other source of energy. No pretension elastic springs or compressed source of the energy is not allowed.

[vi.] The size of the solar cell must not be exceeding with the 15 square inches or 9678 square $\mathrm{mm}$ in size. 


\subsection{Design Approach:}

The mandated problem statement encourages us for the designing of a solar robotic machine that is capable to cover a distance of $1000 \mathrm{~mm}$. in a straight line. The solar powered robots uses the driving motors which use the micro-power for the conversion of the more powerful pulses by using a control circuitry called as the solar engine. Thus, there must be a requirement of a solar engine, which is capable to enhance the performance of the machine in the arena. For a general purpose design implementation, there is a vast availability of the solar engines [6]. The prominent solar engines that, we have tested \{during the implementation phase \} are as follows: The 1381 - based Solar Engine (Miller Engine), FLED based Solar Engine, The T3SE Type 3 Solar Engine, The Power Smart Solar Speeder V2 etc. After testing of these solar engines, we have found that most of the solar engines are based on the Voltage Trigger 1381X IC. These IC's are voltage specific and they come in specific voltage range which lies within 2 to 4.6 Volts. Let's suppose, we are using $1381 \mathrm{~J}$, then it will only trigger at $2.7 \mathrm{~V}$, which results in the limitation of the undesired trigger voltage level. We requires the voltage trigger level according to the arena specifications that must be capable to obtain the minimum time for the crossing of the 1 meter distance in the arena. Thus there must be a need of a solar engine in which the voltage trigger level can be set according to our requirement.

Therefore, we approached towards the Relaxation Oscillator circuit which provides an excellent solution to the problem specified. The best part is that, it even didn't require the $1381 \mathrm{X}$ series IC ( rarely available in India ) and at the same time the desired trigger level can be varied according to the design requirement. The design requirement could be compensate by changing the number of $1 \mathrm{~N} 4148$ fast switching diodes that are connected in series. Also, the circuit involves the basic components of electronics that are easily available in the market.

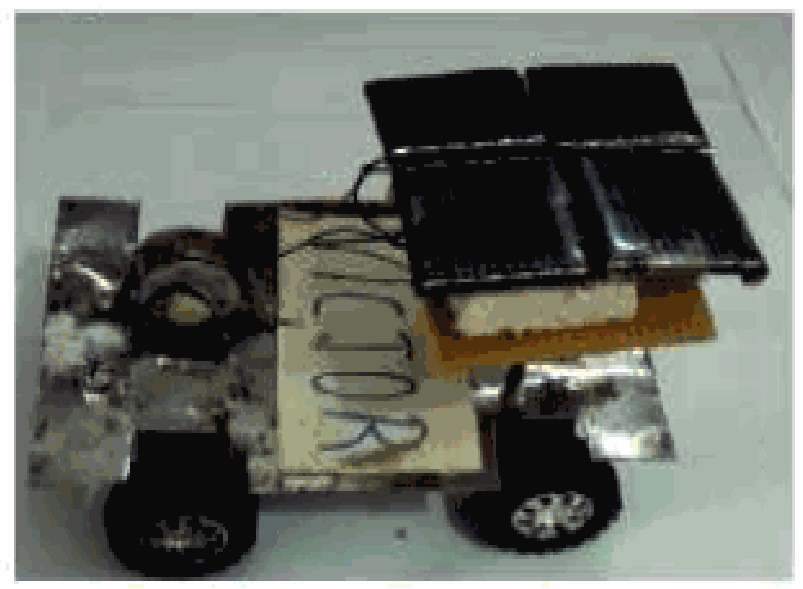

Figure 3.0 Demonstration of Photo-roller designed for nexus roller competition.

\subsection{Working of Relaxation Oscillator:}

This design is based on the application of the relaxation Oscillator as a solar engine. This relaxation oscillator worked as a back-bone of the solar machined mechanism.

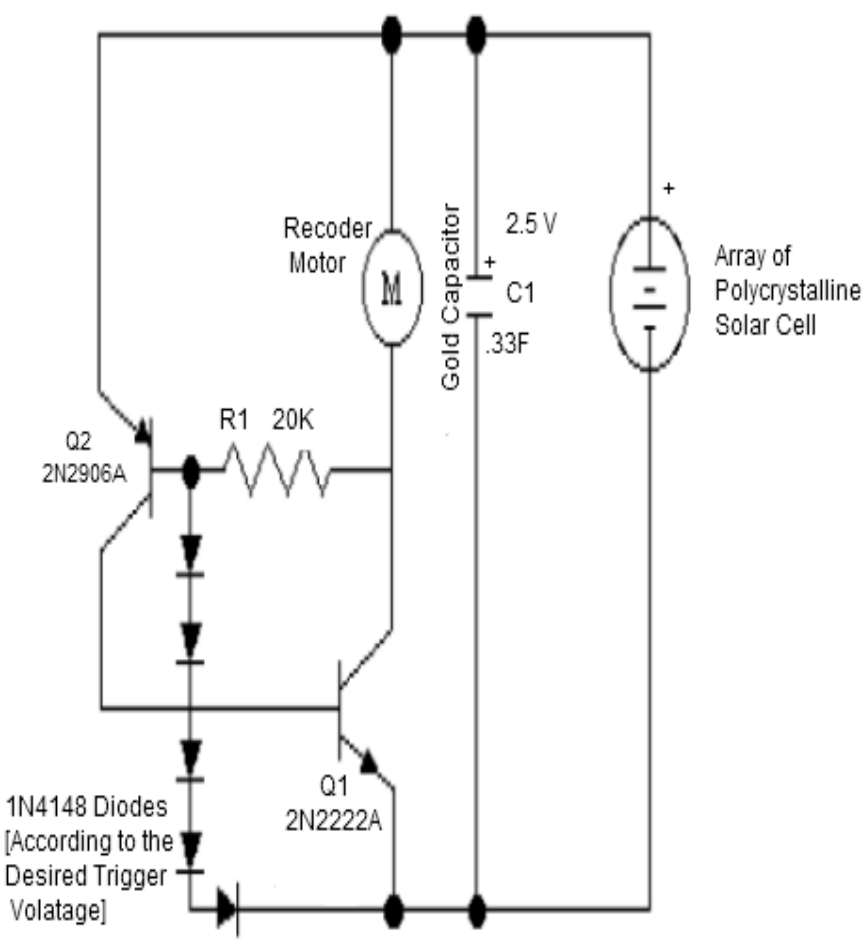

Figure 4. Demonstration of the Circuit Diagram of the relaxation Oscillator used as a solar engine in our Machine: - Photo-Racer.

As, we have discussed previously, the design is based on the application of the relaxation oscillator as the solar engine. The Gold capacitor is implemented as a storage capacitor. Although it takes more time to charge, in spite of that the engine shoots like a bullet. The storage capacitor begins to charge due to the current from the solar cell. The Q2 emitter is positive, which is required for the conduction within the circuit. But, as the Q2 base becomes positive - from the resistor through the motor. At that time the current cannot flow through the Q2 collector to Q2 emitter. In order for the concurrent flow of the current from the Q1 emitter towards the Q1 collector. At that time the Q1 base must be positive while the Q1 collector must be negative. As the voltage of capacitor reaches the trigger voltage of $1 \mathrm{~N} 4148$ diodes that are forward biased. Thus at that time the current flows through the diodes. The current flowing through the diodes establishes a source current from Q2 base to Q2 emitter.

This in turn causes a greater current flow from Q2 collector to the Q2 emitter and allows the Q1 base to check the positivity of the circuit. The current flowing from the Q2 collector to Q2 emitter causes a similar flow of current (within Q1 ) from Q1 emitter to Q1 base, since it is evident that the Q1 base is now positive. This small amount of current flows through the Q1 emitter to the Q1 base and induces a large flow of current from the Q1 emitter to Q2 collector. The flowing current through Q1 emitter to the Q1 collector must pass through the motor and causing it to go spin. The voltage across capacitor falls below the trigger point of diodes. The base of Q2 feed the 
current by the way of diodes and resistor R1. Thus, when the voltage falls below the diodes trigger point, the diodes stop conducting. But at that point the current continues to flow into the Q2 base and must pass through to Q2 emitter while traveling the way of resistor R1. Thus, keeps Q2 on, which in turn Q1 ON and allows the motors to run. This flow is continuous until the motor resistance / load creates a high resistance that essentially halts the flow cycle.

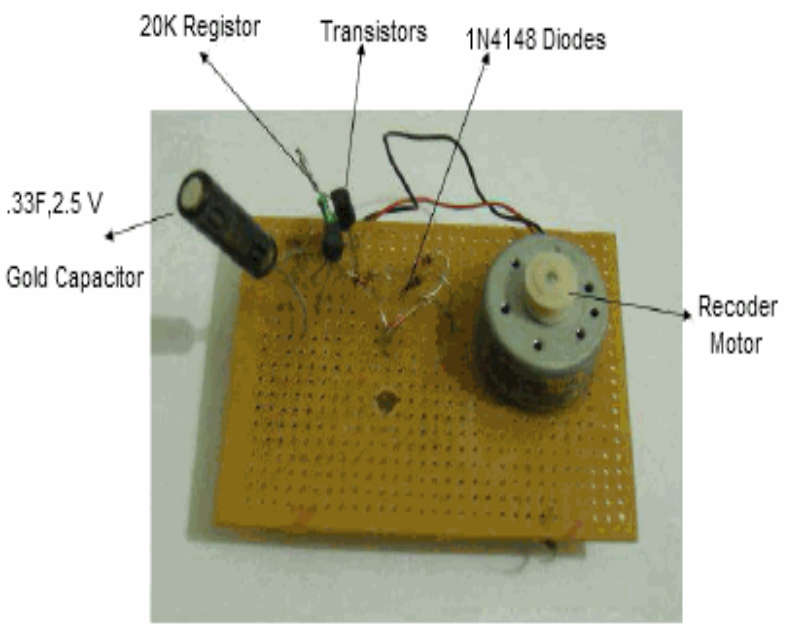

Figure 5. Demonstration of the PCB Design of solar machine: Photo-Racer

*Obstacles:--There are four obstacles that are available in the arena which are painted with the black color and fixed in the four symmetric locations.

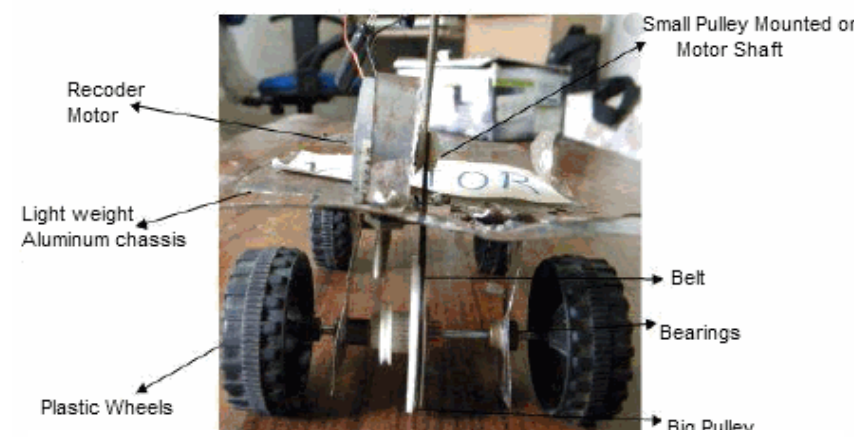

Figure 6. Demonstration of Design Mechanism of our solar machine: Photo-Racer.

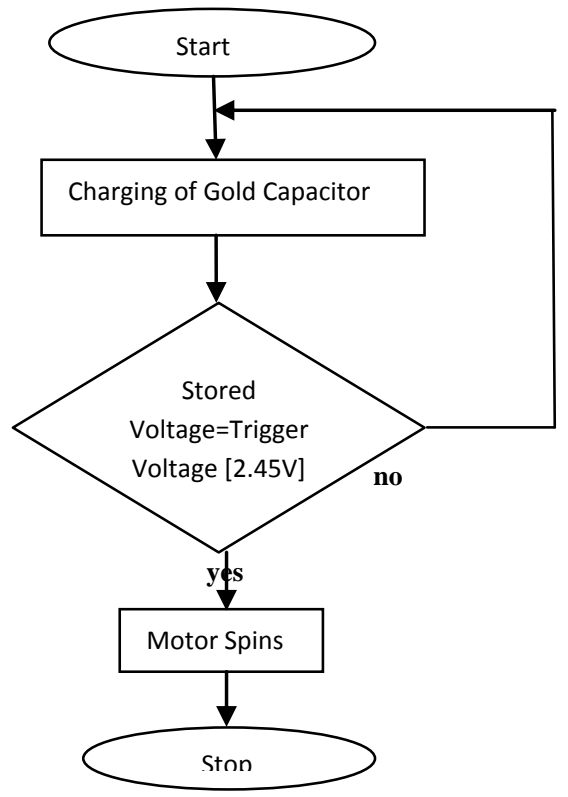

Graph 1. Machine Order Process of execution w.r.t. before and after charging of gold capacitor.

\section{ANALYTICAL \& GRAPHICAL} RESULTS:

The following analytical and graphical results were obtained during the analysis of the solar-robot. These results have been shown below:-

[a.] Analytical Results:

Table 1. Analytical Results in terms of capacitor voltage and time in seconds.

\begin{tabular}{|l|l|l|l|}
\hline Time [sec] & \multicolumn{1}{|c|}{$\begin{array}{c}\text { Capacitor Voltage } \\
{[\text { volt] }}\end{array}$} & $\begin{array}{l}\text { Time } \\
{[\mathbf{s e c}]}\end{array}$ & $\begin{array}{l}\text { Capacitor } \\
\text { Voltage[v] }\end{array}$ \\
\hline 0 & 0 & 11 & 2.14 \\
\hline 1 & 0.21 & 12 & 2.23 \\
\hline 2 & 0.43 & 13 & 2.36 \\
\hline 3 & 0.7 & 14 & 2.45 \\
\hline 4 & 1.02 & 15 & 1.89 \\
\hline 5 & 1.26 & 16 & 1.75 \\
\hline 6 & 1.44 & 17 & 1.53 \\
\hline 7 & 1.53 & 18 & 1.35 \\
\hline 8 & 1.68 & 19 & 1.21 \\
\hline 9 & 1.79 & & \\
\hline 10 & 1.95 & & \\
\hline
\end{tabular}


Table2. Demonstration of the Analytical Results of the testing of Designed solar robot.

\begin{tabular}{|l|l|l|l|}
\hline Time[Sec] & $\begin{array}{c}\text { Current } \\
{[\mathbf{m A}]}\end{array}$ & $\begin{array}{c}\text { Time } \\
{[\text { Sec] }}\end{array}$ & $\begin{array}{c}\text { Current } \\
{[\mathbf{m A}]}\end{array}$ \\
\hline 0 & 0 & 8 & 20 \\
\hline 1 & 20 & 9 & 20 \\
\hline 2 & 20 & 10 & 20 \\
\hline 3 & 20 & 11 & 20 \\
\hline 4 & 20 & 12 & 20 \\
\hline 5 & 20 & 13 & 20 \\
\hline 6 & 20 & 14 & 20 \\
\hline 7 & 20 & & \\
\hline
\end{tabular}

[b.] Graphical Results: The various graphs have been drawn on the basis of the above specified analytical results.

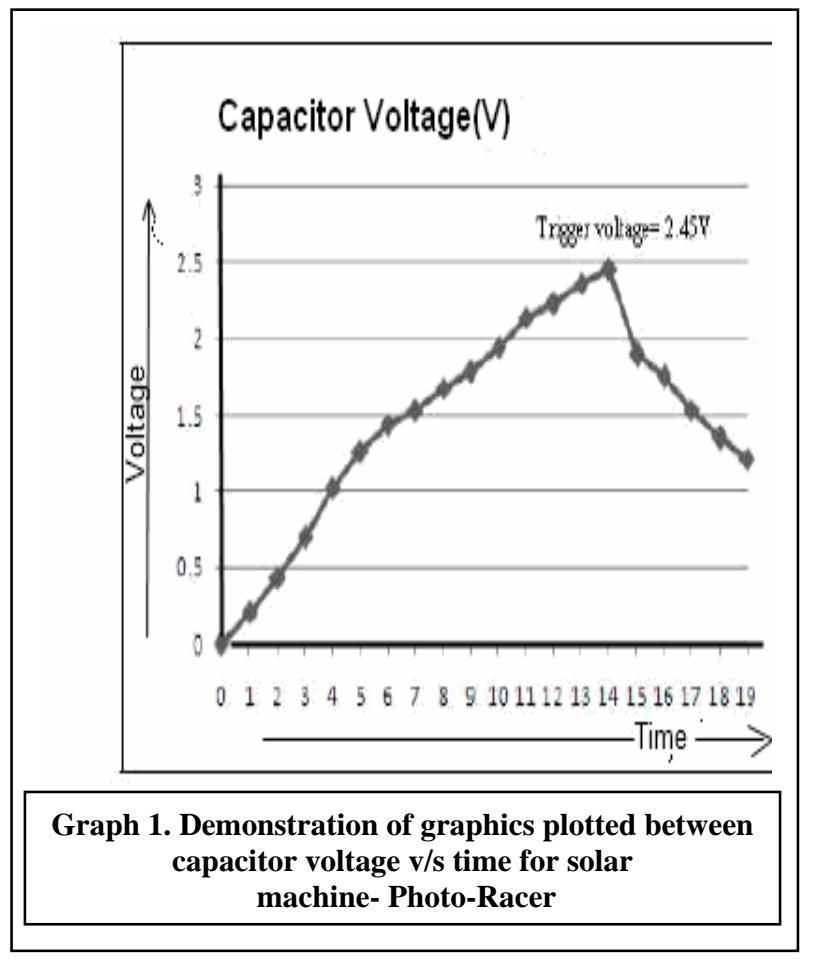

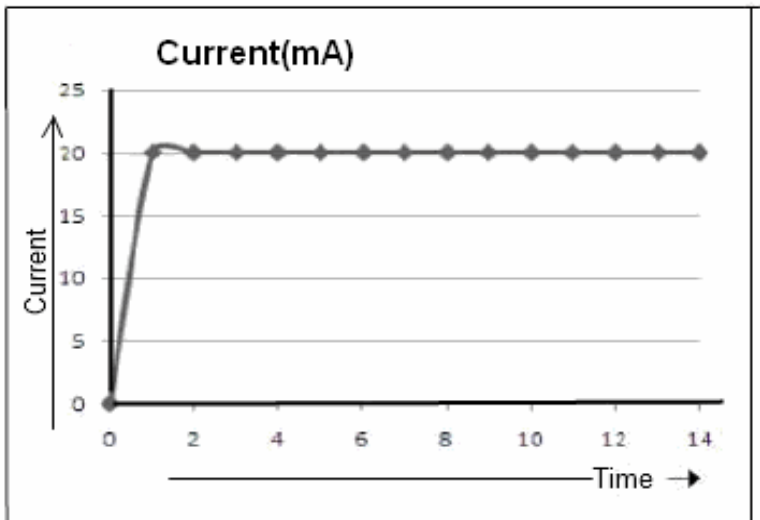

Graph 2. Demonstration of plotted graph that shows the charging current for solar machine -

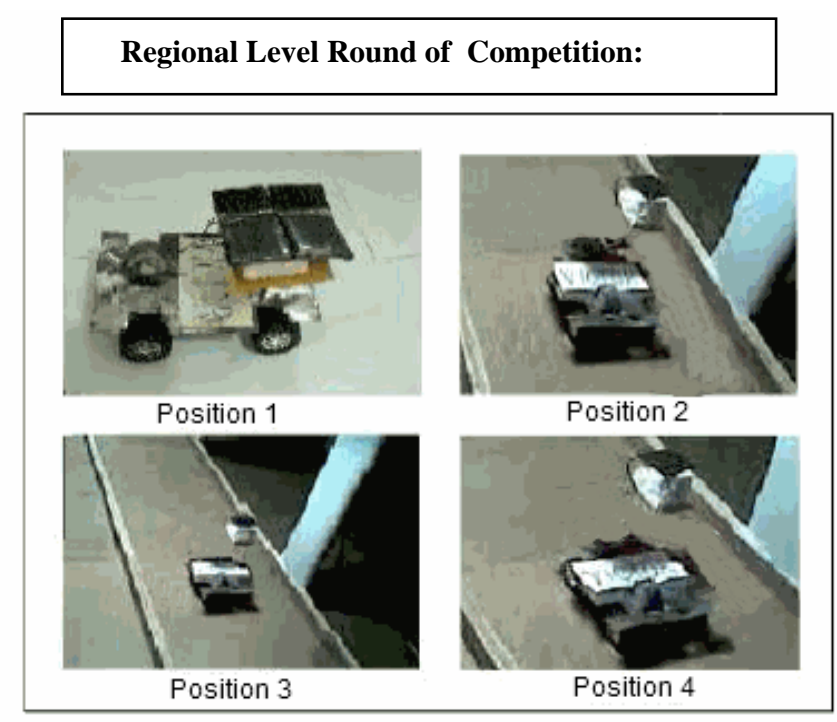

Figure 8. Demonstration of Initial, Mid and Final Position of photo-roller in Nexus Arena.

\section{CONCLUSION \& FUTURE WORK}

This design is the milestone of the light-seeking behavior of the solar robot. The rigorous testing and validation of the circuit was carried out in a comprehensive manner. The applications of the gold capacitor in relaxation oscillator provide us an unbeatable lead in the arena of the Nexus Photo-roller Competition. The student research is always being appreciated by every institution and we think that the student design competition is a good initiative in this direction. It provides them the clarity of concept taught and develops the habit of analytical thinking. Before taking the initiative of designing of Solar Robotics machine a student had to work very hard over the fundamentals of Electronics. In future work, we will extend our approach for the designing of the Biomimetic Underwater Robots, which are biologically inspired robots that exhibit the robust Performance. 


\section{REFERENCES:}

[1] Siddarth Jain, "PhotoChrome: Asia's Largest Techfest Award Winning Solar Robot" in Proceeding of International Conference on Recent Trends in Information, Telecommunication and Computing, March 2010.

[2] Hasslacher, B., Tilden, M. W., "Living Machines", Robotics and Autonomous Systems: The Biology and Technology of Intelligent Autonomous Agents. Editor: L. Steels. Elsevier Publishers, Spring 1995. (LAUR- 942636).

[3] “Look Back- Asia's Largest Technology Extravaganza IIT Bombay Tech fest", available on www .tech fest .org

[4] Asia's Largest, Tech-fest Blog available on www.techfest.org.

[5] Anthony Green and Christophe Jehoulet, The NonBattery - The Potential role of Super Capacitors in Standby Power Applications.
[6] Mr. Marvit's, "Biologically Plausible Dynamic Artificial Neural Networks Reviewed, in Neuron Digest Vol. 11, issue 13, page(s)12-18.

[7] Panasonic Electronic Double Layer Capacitor "Gold Capacitor", Panasonic Industrial Company Production Management Department year 2004.

[8] Solar cell information available on URL http://www.solarbotics.com /products scc3733/resources. 